\title{
A REMARK ON A RESULT OF XIA CHEN
}

\author{
KHOA LÊ
}

\begin{abstract}
We consider the parabolic Anderson model which is driven by a Gaussian noise fractional in time and having certain scaling property in the spatial variables. Recently, Xia Chen has obtained exact Lyapunov exponent for all moments of positive integer orders. In this note, we explain how to extend Xia Chen's result for all moments of order $p$, where $p$ is any real number at least 2 .
\end{abstract}

\section{INTRODUCTION}

The parabolic Anderson model takes the form

$$
\partial_{t} u_{\lambda}-\frac{\Delta}{2} u_{\lambda}=\sqrt{\lambda} u \dot{W}
$$

where $t \geq 0, x \in \mathbb{R}^{d}, \lambda>0$ and $\dot{W}$ is a centered generalized Gaussian field. The covariance of $\dot{W}$ is given by

$$
\mathbb{E}[\dot{W}(t, x) \dot{W}(s, y)]=\gamma_{0}(t-s) \gamma(x-y),
$$

where $\gamma_{0}$ is locally integrable and $\gamma$ is positive definite. The parameter $\lambda$ represents the intensity of the noise. We assume throughout the note that the initial datum of (1) is the constant 1 . We denote by $\mu$ the spectral measure of $\gamma$. Under Dalang's condition

$$
\int_{\mathbb{R}^{d}} \frac{\mu(d x)}{1+|x|^{2}}<\infty
$$

the equation (1) has a unique random field solution $\left\{u_{\lambda}(t, x) ; t \geq 0, x \in \mathbb{R}^{d}\right\}$ such that

$$
u_{\lambda}(t, x)=1+\sqrt{\lambda} \int_{0}^{t} \int_{\mathbb{R}^{d}} p_{t-s}(x-y) u_{\lambda}(s, y) W(d s, d y) .
$$

It is known that $u_{\lambda}(t, x)$ has finite moment of all positive orders. We refer to [3] for a proof of these results. In addition, the moments of natural order can be expressed explicitly through Key words and phrases. parabolic Anderson, Feynman-Kac's representation, hypercontractivity . 
a Feynman-Kac-type formula (c.f. [3,4]), namely

$$
\mathbb{E} u_{\lambda}^{n}(t, x)=\mathbb{E} \exp \left\{\lambda \sum_{1 \leq j<k \leq n} \int_{0}^{t} \int_{0}^{t} \gamma_{0}(s-r) \gamma\left(B_{j}(s)-B_{k}(r)\right) d s d r\right\}
$$

where $B_{1}, \ldots, B_{n}$ are independent $d$-dimensional Brownian motions. We assume the following scaling condition

(S) There exist $\alpha_{0} \in[0,1)$ and $\alpha \in(0,2)$ such that $\gamma_{0}(t)=|t|^{-\alpha_{0}}$ and $\gamma(c x)=c^{-\alpha} \gamma(x)$ for all $x \in \mathbb{R}^{d}$ and all positive numbers $c$.

It is shown by Xia Chen in [2] under conditions (S) and (D) that for every $x \in \mathbb{R}^{d}$ and every integer $n \geq 1$,

$$
\lim _{t \rightarrow \infty} t^{-\frac{4-\alpha-2 \alpha_{0}}{2-\alpha}} \log \mathbb{E} u_{\lambda}^{n}(t, x)=n\left(\frac{n-1}{2}\right)^{\frac{2}{2-\alpha}} \mathcal{E}(\lambda)
$$

where $\mathcal{E}(\lambda)$ is the variation

$$
\mathcal{E}(\lambda)=\sup _{g \in \mathcal{A}_{d}}\left\{\lambda \int_{0}^{1} \int_{0}^{1} \int_{\mathbb{R}^{d} \times \mathbb{R}^{d}} \frac{\gamma(x-y)}{|s-r|^{-\alpha_{0}}} g^{2}(s, x) g^{2}(r, y) d x d y d r d s-\frac{1}{2} \int_{0}^{1} \int_{\mathbb{R}^{d}}\left|\nabla_{x} g(s, x)\right|^{2} d x d s\right\} .
$$

In the above expression, $\mathcal{A}_{d}$ is the class of functions defined as

$$
\mathcal{A}_{d}=\left\{g: g(s, \cdot) \in W^{1,2}\left(\mathbb{R}^{d}\right) \text { and } \int_{\mathbb{R}^{d}} g(s, x) d x=1 \quad \forall 0 \leq s \leq 1\right\}
$$

Under scaling assumption $(\mathrm{S})$, we always have

$$
\mathcal{E}(\lambda)=\lambda^{\frac{2}{2-\alpha}} \mathcal{E}(1)
$$

Our main purpose in this note is to show that (4) holds for any real numbers $n \geq 2$.

Theorem 1. For every $x \in \mathbb{R}^{d}$ and every real number $p \geq 2$, we have

$$
\lim _{t \rightarrow \infty} t^{-\frac{4-\alpha-2 \alpha_{0}}{2-\alpha}} \log \mathbb{E} u_{\lambda}^{p}(t, x)=p\left(\frac{p-1}{2}\right)^{\frac{2}{2-\alpha}} \mathcal{E}(\lambda) .
$$

We note that precise long term asymptotic for moments of real orders have not been obtained in literature. The main difficulty is the lack of moment formulas. The contribution 
of the current paper is a novelty method to extend long term asymptotic for moments of natural orders to those of real orders.

\section{ProOF}

We present the proof of Theorem 1 in the current section. We first observe that in the proof of the lower bound of (4) in [2], we can replace $n$ by any real number $n>1$. Therefore, we always have

$$
\liminf _{t \rightarrow \infty} t^{-\frac{4-\alpha-2 \alpha_{0}}{2-\alpha}} \log \mathbb{E} u_{\lambda}^{p}(t, x) \geq p\left(\frac{p-1}{2}\right)^{\frac{2}{2-\alpha}} \mathcal{E}(\lambda), \text { for all } p>1
$$

On the other hand, Xia Chen's proof of the upper bound of (4) relies heavily on the Feynman-Kac moment representation (3), which is meaningful only for moments of positive integer orders. To obtain the upper bound of (7) which is valid for any real number $p \geq 2$, the key observation is the following inequality, proved in [5].

Lemma 2. For every $q \geq p>1$, we have

$$
\left\|u_{\frac{p-1}{q-1} \lambda}(t, x)\right\|_{L^{q}(\Omega)} \leq\left\|u_{\lambda}(t, x)\right\|_{L^{p}(\Omega)}
$$

for every $t>0$ and $x \in \mathbb{R}^{d}$.

Since this inequality is implicit in [5], we reproduce the proof here.

Proof. Let $\left\{P_{\tau}\right\}_{\tau \geq 0}$ denote the Ornstein-Uhlenbeck semigroup in the Gaussian space associated with the noise $W$. For a bounded measurable function $f$ on $\mathbb{R}^{\mathfrak{H}}$, we have the following Mehler's formula

$$
P_{\tau} f(W)=\mathbb{E}^{\prime} f\left(e^{-\tau} W+\sqrt{1-e^{-2 \tau}} W^{\prime}\right),
$$

where $W^{\prime}$ an independent copy of $W$, and $\mathbb{E}^{\prime}$ denotes the expectation with respect to $W^{\prime}$. For each $\tau \geq 0$, let $u_{\tau, \lambda}$ be the solution to equation (1) driven by the space-time Gaussian 
field $\sqrt{\lambda}\left(e^{-\tau} W+\sqrt{1-e^{-2 \tau}} W^{\prime}\right)$, with initial condition $u_{0}=1$. That is,

$$
\begin{aligned}
u_{\tau, \lambda}(t, x)= & 1 \\
& +\sqrt{\lambda} \int_{0}^{t} \int_{\mathbb{R}^{d}} p_{t-s}(x-y) u_{\tau, \lambda}(s, y)\left(e^{-\tau} W(d s, d y)+\sqrt{1-e^{-2 \tau}} W^{\prime}(d s, d y)\right) .
\end{aligned}
$$

From Mehler's formula we see that $P_{\tau} u_{\lambda}=\mathbb{E}_{W^{\prime}}\left[u_{\tau, \lambda}\right]$ satisfies the equation

$$
P_{\tau} u_{\lambda}(t, x)=1+\sqrt{\lambda} e^{-\tau} \int_{0}^{t} \int_{\mathbb{R}^{d}} p_{t-s}(x-y) P_{\tau} u_{\lambda}(s, y) W(d s, d y) .
$$

In other words, $P_{\tau} u_{\lambda}$ is another solution of (1) with $\lambda$ being replaced by $\lambda e^{-2 \tau}$. By uniqueness, we conclude that $P_{\tau} u_{\lambda}=u_{e^{-2 \tau} \lambda}$. On the other hand, it is well-known that the OrnsteinUhlenbeck semigroup verifies the following hypercontractivity inequality

$$
\left\|P_{\tau} f\right\|_{q(\tau)} \leq\|f\|_{p}
$$

for all $1<p<\infty$ and $\tau \geq 0$, where $q(\tau)=1+e^{2 \tau}(p-1)$. Hence, applying the hypercontractivity property to our situation yields

$$
\left\|u_{e^{-2 \tau} \lambda}(t, x)\right\|_{q(\tau)} \leq\left\|u_{\lambda}(t, x)\right\|_{p}
$$

for all $t>0, x \in \mathbb{R}^{d}$ and $\tau \geq 0$. The result follows by choosing $\tau$ so that $q(\tau)=q$, that is $e^{-2 \tau}=\frac{p-1}{q-1}$

Turning back to the upper bound in (7), let us choose $p=2$ in (9) to obtain

$$
\left\|u_{\frac{\lambda}{q-1}}(t, x)\right\|_{L^{q}(\Omega)} \leq\left\|u_{\lambda}(t, x)\right\|_{L^{2}(\Omega)}
$$

for all $q \geq 2$. We can apply (4) to get for all $q \geq 2$ and $\lambda>0$

$$
\limsup _{t \rightarrow \infty} t^{-\frac{4-\alpha-2 \alpha_{0}}{2-\alpha}} \log \mathbb{E} u_{\frac{\lambda}{q-1}}^{q}(t, x) \leq 2^{-\frac{2}{2-\alpha}} \mathcal{E}(\lambda)
$$

which, by the scaling relation (6), is equivalent to the upper bound for (7). 


\section{Conclusion}

Using hypercontractivity of Ornstein-Uhlenbeck semigroup, precise long term asymptotic of $\mathbb{E} u^{p}(t, x)$ is obtained for all real numbers $p \geq 2$. We conclude our paper with the following remarks.

(a) The lower bound (8) holds for all $p>1$. Base on this evident, we conjecture that (7) holds for every real number $p \geq 1$ (note that the case $p=1$ is trivial).

(b) From the expression (7), the inequality (9) is indeed an equality in the limit $t \rightarrow \infty$. The situation is quite different if $W$ is a space-time white noise. In fact, in this case, it is proved in [1] that for every integer $n \geq 1$,

$$
\lim _{t \rightarrow \infty} \frac{1}{t} \log \mathbb{E} u_{\lambda}^{n}(t, x)=\frac{n\left(n^{2}-1\right) \lambda^{2}}{24}
$$

It follows that inequality (9) is strict in the limit $t \rightarrow \infty$. Therefore, our method can not be directly applied in this case. It is, however believed in physics literature that (12) holds for any real number $n>0$.

Acknowledgment: The author was supported by the NSF Grant no. 0932078 000, while he visited the Mathematical Sciences Research Institute in Berkeley, California, during the Fall 2015 semester. The author thanks Jingyu Huang for helpful discussions and encouragement.

\section{REFERENCES}

[1] X. Chen, Precise intermittency for the parabolic Anderson equation with an $(1+1)$-dimensional time-space white noise, Ann. Inst. Henri Poincaré Probab. Stat. 51 (2015), no. 4, 1486-1499. 个5

$[2]$ _ Moment asymptotics for parabolic Anderson equation with fractional time-space noise: in Skorohod regime, Annales de l'Institut Henri Poincare (to appear). $\uparrow 2,3$

[3] Y. Hu, J. Huang, D. Nualart, and S. Tindel, Stochastic heat equations with general multiplicative Gaussian noises: Hölder continuity and intermittency, Electron. J. Probab. 20 (2015), no. 55, 50. 个1, 2

[4] Y. Hu, D. Nualart, and J. Song, Feynman-Kac formula for heat equation driven by fractional white noise, Ann. Probab. 39 (2011), no. 1, 291-326. ^2

[5] J. Huang, K. Lê, and D. Nualart, Large time asymptotics for the parabolic Anderson model driven by spatially correlated noise, Annales de l'Institut Henri Poincare (to appear). $\uparrow 3$ 
Mathematical Sciences Research Institute, Berkeley, California, USA

E-mail address: khoa.le@ucalgary.ca 